\title{
Flutuação populacional de muscídeos (Diptera, Muscidae) simbovinos e sua distribuição sobre o corpo do gado de leite, em Capão do Leão, RS, Brasil
}

\author{
Fluctuation of symbovine muscids (Diptera, Muscidae) and their distribution on the bodies of \\ dairy cattle in Capão do Leão, RS, Brazil
}

\author{
Cristine Ramos Zimmer ${ }^{\mathrm{I}, \text { II }^{\star}}$ Dani Furtado Araújo ${ }^{\mathrm{II}}$ Paulo Bretanha Ribeiro,II
}

\section{RESUMO}

Este estudo teve como objetivo conhecer as espécies de muscídeos simbovinos e a área de eleição destas sobre o corpo do bovino de leite. Também estimou-se a flutuação populacional das espécies de moscas, relacionando-a com as médias mensais de temperatura e umidade relativa, bem como a precipitação pluviométrica em Capão do Leão, Rio Grande do Sul, Brasil. Para estimar a sazonalidade e a localização sobre bovinos, foram examinadas 20 vacas leiteiras a cada 30 dias, no período de abril/2007 a março/2008, conforme as divisões zootécnicas por meio da observação direta dos dípteros. Os muscídeos simbovinos verificados foram: Haematobia irritans, espécie mais abundante e encontrada em maior frequência na costela/no flanco, seguida por Stomoxys calcitrans, com maior ocorrência nos membros dianteiros e traseiros e Musca domestica, na cabeça, no ventre e na costela/ no flanco dos bovinos. A sazonalidade foi influenciada principalmente pela temperatura, exercendo forte impacto negativo em situações extremas. Na temperatura média menor ou igual a $12,7^{\circ} \mathrm{C}$ e na temperatura acima de $22,5^{\circ} \mathrm{C}$, não houve observação de dípteros.

Palavras-chave: Haematobia irritans, Stomoxys calcitrans, Musca domestica, sazonalidade, bovino de leite.

\section{ABSTRACT}

This study aimed to describe the symbovine species and which area of the dairy cattle body these flies selectes. In addition the fluctuation of these species population was estimated relative to the average monthly temperature, humidity and rainfall in Capão do Leão, Rio Grande do Sul, Brazil. To estimate seasonality and location on the cattle, 20 dairy cattle were examined through flies' direct observation every 30 days in the period from April/2007 to March/2008 according to the animal zootechnical divisions. The symbovine muscids checked were: Haematobia irritans, the most abundant species and found in greater frequency on the rib/flank, followed by Stomoxys calcitrans, with higher occurrence on the front and rear legs, and Musca domestica, on the head, belly and rib/ flank. Seasonality was mainly influenced by temperature which had a strong and negative impact in extreme situations. There was no observation of flies with average temperature less than or equal to $12.7^{\circ} \mathrm{C}$ and above $22.5^{\circ} \mathrm{C}$.

Key words: Haematobia irritans, Stomoxys calcitrans, Musca domestica, seasonality, dairy cattle

\section{INTRODUÇÃO}

As principais espécies de Muscidae que ocorrem em agroecossistemas rurais com produção de animais são: Musca domestica Linnaeus, 1758, Stomoxys calcitrans (Linnaeus, 1758) e Haematobia irritans (Linnaeus, 1758) (MARCHIORI et al., 2001). Esses dípteros são atraídos pelas fezes dos animais, pela disponibilidade de alimentos e pelo abrigo aos adultos.

Quando as populações de dípteros ultrapassam o nível de dano econômico, ocasionam alguns problemas em razão do hábito que possuem de defecar e regurgitar sobre as superfícies, além de veicular patógenos (AXTELL \& ARENDS, 1990).

A presença de dípteros hematófagos associados ao gado leiteiro causa consideráveis prejuízos na produtividade (CAMPBELL et al., 2001).

IPrograma de Pós-graduação em Fitossanidade, Universidade Federal de Pelotas (UFPel), Pelotas, RS, Brasil.

"Laboratório de Biologia e Taxonomia de Insetos, Departamento de Microbiologia e Parasitologia, Instituto de Biologia, UFPel. Campus Universitário, CP 354, 96015-560, Capão do Leão, RS, Brasil. E-mail: crzimmerbio@yahoo.com.br. *Autor para correspondência. 
GUIMARÃES (1984) cita que a elevada incidência de S. calcitrans, popularmente conhecida como "moscados-estábulos”, em determinadas áreas, pode acarretar perdas de 20 a $60 \%$ na produção de leite. Os danos atribuídos à $\boldsymbol{H}$. irritans, comumente chamada de "mosca-do-chifre", com respeito ao rebanho nacional de bovinos, foi estimada em US\$ 150 milhões (GRISI et al., 2002).

A flutuação e a composição das populações de muscóideos sinantrópicos são definidas por meio de fatores bióticos e abióticos (NUORTEVA, 1963; DAJOZ, 1983). A flutuação populacional é uma alternativa de avaliação das populações no tempo e no espaço, pela impossibilidade de verificação da densidade absoluta de insetos.

Este trabalho foi realizado para conhecer as espécies de muscídeos simbovinas e a área de eleição sobre o corpo de bovino de leite. Também estimou-se a flutuação populacional das espécies de moscas, relacionando-as com as médias mensais de temperatura, umidade relativa e precipitação pluviométrica em Capão do Leão, Rio Grande do Sul, Brasil.

\section{MATERIAL E MÉTODOS}

O estudo foi conduzido no Centro Agropecuário da Palma/Universidade Federal de Pelotas (UFPel), no Município de Capão do Leão, localizado na Planície Costeira do Rio Grande do Sul, cujas coordenadas geográficas são $31^{\circ} 52^{\prime} 00^{\prime \prime}$ de latitude sul e $52^{\circ} 21^{\prime} 24^{\prime \prime}$ de longitude oeste e altitude de 30 metros.

Foram utilizadas 20 vacas leiteiras das raças Holandesa, Jersey e mestiças, criadas em regime de semiconfinamento, sem receber tratamento de produtos que interferissem na população de adultos das moscas. Os animais foram examinados a cada 30 dias, no período de abril de 2007 a março de 2008. No momento em que os animais eram ordenhados, em torno das 16 horas, foi realizada a observação direta de moscas sobre a metade direita dos bovinos, conforme as divisões propostas por RIBEIRO et al., (1989), (Figura 1), e o número de insetos observados foram multiplicado por dois para obtenção do número total por animal (GARCÍA et al., 2001).

A frequência populacional dos dípteros simbovinos foi verificada por meio da média de exemplares por espécie e localização no corpo do bovino. Estimou-se a flutuação populacional de cada espécie de mosca, por meio da média mensal por bovino, associando-se as variáveis climáticas: temperatura, umidade relativa e precipitação pluviométrica. Os dados climáticos foram fornecidos pela Estação Agroclimatológica de Pelotas, localizada em Capão do Leão, cujas coordenadas geográficas são $31^{\circ} 52^{\prime} 00^{\prime \prime}$ de latitude sul e $52^{\circ} 21^{\prime} 24^{\prime \prime}$ de longitude oeste e altitude de 13,24 metros.

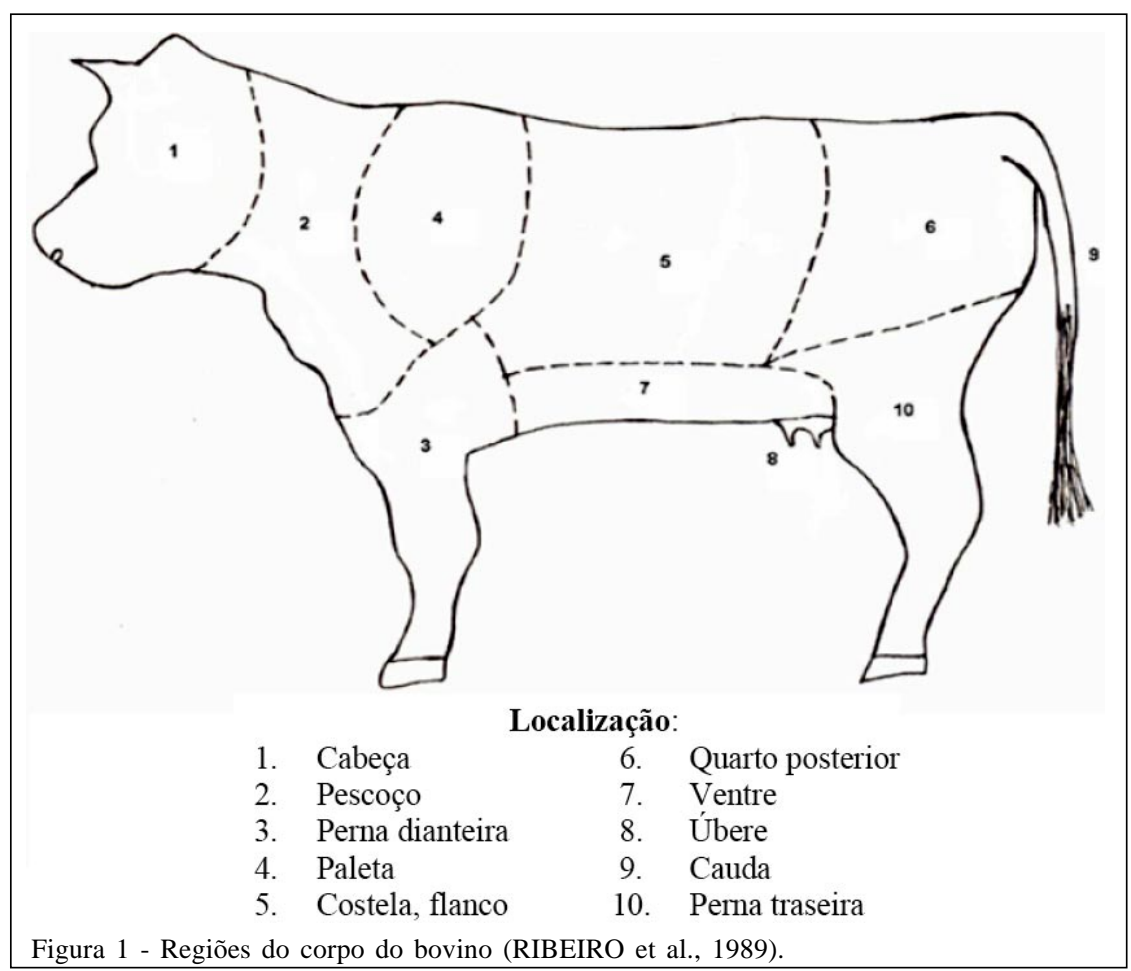

Ciência Rural, v.40, n.3, mar, 2010. 


\section{RESULTADOS}

Durante o período experimental, foi observado o total de 10.444 muscídeos sobre bovinos de leite, pertencentes à família Muscidae. A espécie mais abundante foi $\boldsymbol{H}$. irritans, com frequência de $72,71 \%$ (7.594), seguida por $\boldsymbol{S}$. calcitrans, com $16,90 \%$ (1.765), ambas as espécies hematófagas, enquanto que M. domestica, lambedora, foi a espécie de menor ocorrência, com apenas 10,39\% (1.085) dos espécimes capturados (Tabela 1).

Em abril, a média verificada dos dípteros foi de 1.874 insetos, em que $\boldsymbol{H}$. irritans foi a espécie mais abundante (49,09\%), seguida por $\boldsymbol{S}$. calcitrans $(30,31 \%)$ e $\boldsymbol{M}$. domestica (20,60\%), tendência que se manteve durante todo o período experimental (Figura 2).

No período de maio a agosto de 2007, a população de dípteros foi ausente sobre o gado (Figura 2). Esse comportamento pode ser explicado pelas baixas temperaturas médias mensais, que variaram de 9,9 a $12,7^{\circ} \mathrm{C}$ (Figura 3A), condições adversas aos dípteros, caracterizando o período desfavorável para ocorrência de moscas simbovinas.

No mês de setembro, os insetos voltaram a infestar o gado (Figura 2), permanecendo sobre os animais até março, caracterizando o período favorável de ocorrência de moscas. As condições climáticas favoreceram a presença dos muscídeos sobre os bovinos, quando as temperaturas médias mensais variaram de 16,8 a $22,5^{\circ} \mathrm{C}$. Os picos populacionais dos dípteros no gado de leite (Figura 2) ocorreram respectivamente em março (3.213), novembro (2.047), abril (1.874), fevereiro(1.641)e dezembro(1.169), correspondendo aos meses mais quentes do ano (Figura 3).
Em janeiro, foi registrada a temperatura média mensal mais alta do período experimental $\left(23,2^{\circ} \mathrm{C}\right)$ (Figura 3A), e foram registradas a umidade relativa $(75,3 \%)$ e pluviosidade média mensal $(80,4 \%)$ mais baixas durante o período do estudo (Figura 3B). A ação combinada dessas condições possivelmente afetou a ocorrência de muscídeos.

Os três muscídeos simbovinos apresentaram, de forma geral, como área de eleição no corpo do gado de leite, a região da costela/do flanco (região 5), porém cada espécies teve sua preferência particular (Tabela 1). H. irritans foi encontrada com maior frequência na costela/no flanco, seguida pela região do ventre (região 7) e pelas paletas (região 4), das quais a região 5 foi a que apresentou o maior índice $(47,36 \%)$, enquanto que a região da cabeça (região 1$)$ foi a de menor frequência $(0,21 \%)$ (Tabela 1$)$.

Em S. calcitrans, observou-se uma preferência pelos membros anteriores e posteriores. O número de moscas encontradas nessas duas regiões correspondeu a 59,2\% do total, em comparação com as demais regiões (Tabela 1 ).

A maior frequência de $\boldsymbol{M}$. domestica foi verificada na cabeça dos animais (21,66\%), seguida pelo ventre $(21,00 \%)$ e pela costela/pelo flanco $(18,70 \%)$ (Tabela 1). A presença da mosca doméstica na região da cabeça pode estar relacionada ao fato de a espécie apresentar aparelho bucal lambedor e provavelmente alimentar-se das secreções oculares e orais dos bovinos.

Tabela 1 - Distribuição de frequência da localização de muscídeos simbovinos sobre bovinos de leite no período de abril de 2007 a março de 2008, no centro agropecuário da Palma/UFPel, em Capão do Leão, RS.

\begin{tabular}{|c|c|c|c|c|c|c|c|c|}
\hline \multirow[b]{2}{*}{ Partes do Bovino } & \multicolumn{2}{|c|}{ Haematobia irritans } & \multicolumn{2}{|c|}{ Stomoxys calcitrans } & \multicolumn{2}{|c|}{ Musca domestica } & \multicolumn{2}{|c|}{$\Sigma$} \\
\hline & $\operatorname{Abs}\left(n^{\circ}\right)$ & Rel (\%) & $\operatorname{Abs}\left(n^{\circ}\right)$ & Rel (\%) & $\operatorname{Abs}\left(n^{\circ}\right)$ & Rel (\%) & $\operatorname{Abs}\left(n^{\circ}\right)$ & Rel (\%) \\
\hline 1. Cabeça & 16 & 0,21 & 48 & 2,72 & 235 & 21,66 & 299 & 2,86 \\
\hline 2. Pescoço & 674 & 8,87 & 78 & 4,42 & 34 & 3,13 & 786 & 7,53 \\
\hline 3. Perna dianteira & 128 & 1,68 & 532 & 30,14 & 96 & 8,84 & 756 & 7,24 \\
\hline 4. Paleta & 1.314 & 17,30 & 112 & 6,34 & 50 & 4,61 & 1.476 & 14,13 \\
\hline 5. Costela/flanco & 3.597 & 47,36 & 224 & 12,69 & 203 & 18,70 & 4.024 & 38,53 \\
\hline 6.Quarto posterior & 270 & 3,55 & 56 & 3,17 & 82 & 7,56 & 408 & 3,91 \\
\hline 7. Ventre & 1.423 & 18,74 & 188 & 10,65 & 218 & 21,00 & 1.829 & 17,51 \\
\hline 8. Úbere & 38 & 0,50 & 0 & 0,00 & 67 & 6,16 & 105 & 1,01 \\
\hline 9. Cauda & 26 & 0,34 & 14 & 0,79 & 40 & 3,69 & 80 & 0,77 \\
\hline 10. Perna traseira & 108 & 1,42 & 513 & 29,06 & 60 & 5,53 & 681 & 6,52 \\
\hline Total & 7.594 & 100,00 & 1.765 & 100,00 & 1.085 & 100,00 & 10.444 & 100,00 \\
\hline
\end{tabular}

Abs: absoluta; Rel: relativa.

Ciência Rural, v.40, n.3, mar, 2010. 


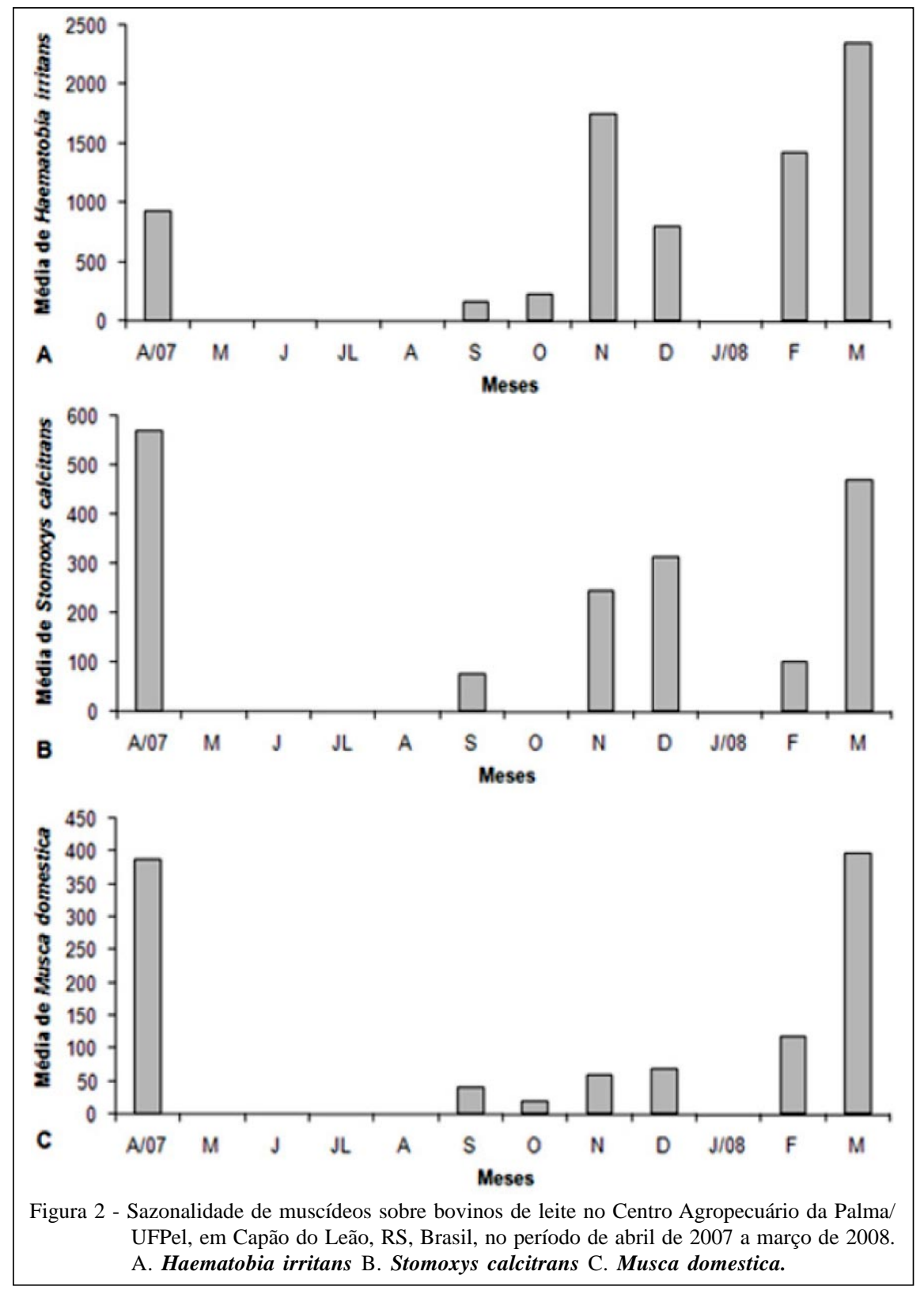

\section{DISCUSSÃO}

A frequência de $\boldsymbol{H}$. irritans verificada em todo período experimental se explica pelo fato de a espécie ser um ectoparasita específico de bovinos e permanecer durante todo o estágio adulto sobre os animais. A mosca-do-chifre é um parasita hematófago contínuo, já que se mantém sobre o hospedeiro dia e noite, alimentando-se 20 a 30 vezes por dia e afastandose do bovino quando busca o bolo fecal recente para realizar a oviposição (GARCÍA et al., 2001).
S. calcitrans é uma espécie hematófaga, oportunista, alimentando-se em uma ampla variedade de hospedeiros, dentre eles os bovinos (ZUMPT, 1973). Realiza a hematofagia uma vez ao dia, permanecendo sobre seus hospedeiros de dois a cinco minutos, tempo suficiente para o repasto sanguíneo (MOON, 2002), razões pela quais foi encontrada em menor abundância, em comparação com a $\boldsymbol{H}$. irritans.

M. domestica, por ser um díptero lambedor e apresentar um comportamento alimentar onívoro, detém de uma vasta diversidade de fontes alimentares (LYSYK \& AXTELL, 1987, MOON, 2002), assim é a 


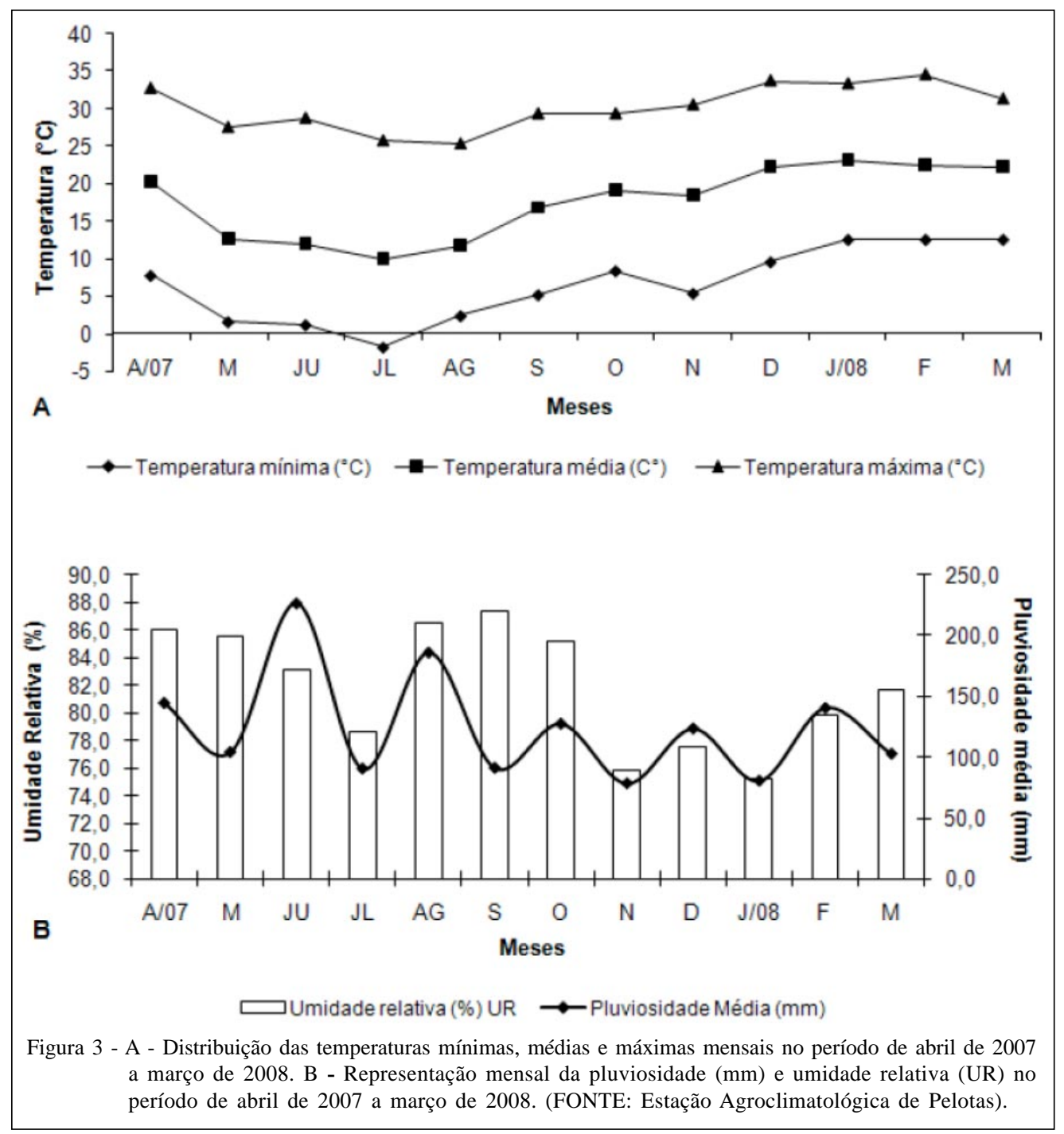

espécie com menor frequência sobre o gado, que vai até esse hospedeiro em busca de secreções corporais.

As variáveis ambientais mais óbvias que confrontam um inseto são as flutuações sazonais na temperatura e umidade. Quando enfrentam adversidades ambientais como condições extremas, as populações de insetos têm como opção disponível para evitar essa condição a fuga comportamental, como, por exemplo, a diapausa, (GULLAN \& CRANSTON, 2007).

O fenômeno de diapausa, em pupas de $\boldsymbol{H}$. irritans, é observado em populações dos trópicos (MENDES \& LINHARES, 1999), que se acentua em climas temperados (LYSYK \& MOON, 1994; 2002). No Sul dos Estados Unidos, THOMAS \& KUNZ (1986) verificaram que pupas de $\boldsymbol{H}$. irritans entram em diapausa no outono e permanecem durante o inverno, começam a emergir no início da primavera, e os picos populacionais são verificados no verão, quando a temperatura e a umidade relativa são mais elevadas. Esse fenômeno também foi observado no México, onde durante o inverno foi constatada ausência de moscas sobre o gado por 60 dias, reaparecendo na primavera (CRUZ-VÁZQUEZ et al, 2000). Durante o período experimental, verificou-se que a permanência dos insetos sobre o gado foi afetada pela temperatura, constatando-se a ausência dos muscídeos em vários meses; numa tentativa de sobrevivência, esses insetos provavelmente podem ter entrado em diapausa.

Especificamente no mês de janeiro de 2008, a alta temperatura e a baixa umidade relativa produziram um impacto negativo sobre as populações de muscídeos, pois o grande desafio dos insetos nessas condições é a obtenção de oxigênio, sem que ocorra a perda de água, o que, devido ao pequeno tamanho

Ciência Rural, v.40, n.3, mar, 2010. 
corpóreo, torna-se difícil (GULLAN \& CRANSTON, 2007). Assim, a ausência de dípteros no período pode ter ocorrido pela diminuição do metabolismo, o que afeta diretamente sua atividade.

De acordo com MOON (2002), no hemisfério Sul, de todos os artrópodes parasitas, $\boldsymbol{H}$. irritans apresenta o maior impacto sobre a saúde e a produtividade de bovinos. As picadas de $\boldsymbol{H}$. irritans são muito doloridas, e a lesão provocada pela hematofagia compromete a qualidade do couro para comercialização. Assim, a presença da $\boldsymbol{H}$. irritans, ainda que em baixos níveis, além de representar redução no ganho de peso dos animais, afetando a produção leiteira, também compromete a comercialização de subprodutos (MOON, 2002; BIANCHIN et al., 2004).

HILLERTON \& BRAMLEY (1986) analisaram o número de $\boldsymbol{H}$. irritans sobre bovinos, observando maior prevalência nas regiões do dorso e do abdome. SCHREIBER \& CAMPBELL (1986) constataram que as regiões com maior número de dípteros foram as regiões do dorso, a escápula, o lado do corpo e o ventre. HONER et al. (1991) relataram a permanência preferencial de $\boldsymbol{H}$. irritans na região costal, na paleta, no ventre e nas pernas. LIMA et al. (2002) também verificaram regiões "interescapular”, "escapular” e "costal”, com maior frequência de dípteros. A similaridade nos resultados deste estudo com os resultados dos estudos supracitados sugere que a preferência dos dípteros por essas regiões nos bovinos ocorreu por esses locais estarem fora do alcance da cabeça e cauda do hospedeiro.

S. calcitrans também apresenta uma picada muito dolorosa, provocando coceira, o que ocasiona estresse nos animais, afetando a capacidade produtiva (MOON, 2002). BRUCE \& DECKER (1958) estudaram a relação entre a quantidade dos dípteros e a redução na produção de leite, verificando que, durante os meses de verão, cada mosca ocasionava uma perda média mensal na produção de $0,65-0,70 \%$ por vaca. No Brasil, GUIMARÃES (1984) cita que, em áreas com elevada incidência de $\boldsymbol{S}$. calcitrans, pode ocorrer um decréscimo de 20 a 60\% na produção leiteira.

Em uma perspectiva econômica, a presença de $\boldsymbol{S}$. calcitrans aumenta os custos da produção de carne porque afeta as exigências de peso ideal para o abate dos bovinos, assim como a lactação das vacas leiteiras (MOON, 2002). Possivelmente, a preferência de $\boldsymbol{S}$. calcitrans pelos membros justifica-se pelo fato de tratar-se de regiões muito vascularizadas, conforme já descrito por BITTENCOURT \& MOYA BORJA (2002).

M. domestica, por apresentar alta mobilidade, entrar em contato com excreções, carcaças, lixo e outros materiais sépticos, apresentar íntima associação com humanos e possuir alto poder reprodutivo, é apontada como importante vetor de patógenos ao homem e outros animais (MENDES \& LINHARES, 1993). Dessa forma, mesmo que $\boldsymbol{M}$. domestica não reduza diretamente o desempenho de bovinos, seu controle é necessário principalmente pela proximidade do agroecossistema rural a áreas urbanas, já que, a não supressão desta mosca envolve a possibilidade da difusão de várias doenças (KEIDING, 1986).

O conhecimento da dinâmica populacional das moscas simbovinas do gado leiteiro nessa região, juntamente com as informações sobre o comportamento dos dípteros, permitirá estabelecer com maior precisão um programa de controle estratégico, que, nesse caso, deveria começar antes de setembro e outubro, quando inicia o aumento das populações de moscas.

\section{CONCLUSÕES}

Diante das condições experimentais deste estudo, pode-se concluir que $\boldsymbol{H}$. irritans foi a espécie mais abundante sobre bovinos de leite, seguida por $\boldsymbol{S}$. calcitrans e $\boldsymbol{M}$. domestica, respectivamente; temperaturas extremas, baixas bem como altas, combinadas com baixa umidade relativa, reduzem as populações de muscídeos simbovinos; $\boldsymbol{H}$. irritans ocorre em maior abundância na região da costela/do flanco, $\boldsymbol{S}$. calcitrans apresenta maior frequência nos membros dianteiros e traseiros, enquanto que $\boldsymbol{M}$. domestica demonstra uma alta frequência na cabeça, no ventre e na costela/no flanco dos bovinos.

\section{REFERÊNCIAS}

AXTELL, R.C.; ARENDS, J.J. Ecology and management of arthropod pests of poultry. Annual Review of Entomology, v.35, p.101-126, 1990. Disponível em: <http://arjournals.annualreviews.org/>. Acesso em: 27 nov. 2009. doi:10.1146/annurev.en.35.010190.000533.

BIANCHIN, I. et al. Efeito da mosca-dos chifres, Haematobia irritans (L.) (Diptera: Muscidae), no ganho de peso de bovinos Nelore. Ciência Rural, v.34, n.3, p.885-890, 2004. Disponível em: <http://www.scielo.br/>. Acesso em: 27 nov. 2009. doi: 10.1590/S0103-84782004000300035

BITTENCOURT, A.J.; MOYA BORJA, G.E. Stomoxys calcitrans (Linnaeu, 1758) (Diptera, Muscidae): preferência por locais do corpo do bovino para alimentação. Revista Brasileira de Zoociências, v.4, p.75-83, 2002.

BRUCE, W.N.; DECKER, G.C. The relationship of stable fly abundance to milk production in dairy cattle. Journal of Economic Entomology, v.51, p.269-274, 1958. 
CAMPBELL, J.B. et al. Effects of stable flies (Diptera: Muscidae) on weight gains of grazing yearling cattle. Journal of Economic Entomology, v.94, p.780-783, 2001. Disponível em: <http://www.bioone.org/doi/abs/>. Acesso em 27 nov. 2009. doi: 10.1603/0022-0493-94.3.780

CRUZ-VÁZQUEZ, C. et al. Distribución anual de Haematobia irritans (L.) (Diptera: Muscidae) en tres establos lecheros de Aguascaliente, México. Veterinária México, v.31, p. 1951992000.

DAJOZ, R. Ecologia geral. 4.ed. Petrópolis: Vozes, 1983. 472p.

GARCíA, C.A. et al. Dinámica populacional de Haematobia irritans em um hato de bovinos de Soto La Marina. Taumalipas, México. Veterinaria México, v.2 p.149-152, 2001.

GRISI, L. et al. Impacto econômico das principais ectoparasitoses em bovinos no Brasil. A Hora Veterinária, v.21, n.1, p.8-10, 2002.

GUIMARÃES, J.H. Mosca dos estábulos - Uma importante praga do gado. Agroquímica Ciba: Geigy, v.23, p.10-14, 1984.

GULLAN, P.J.; CRASTON. Os insetos: um resumo de entomologia. 3.ed. São Paulo: Roca, 2007. 440p.

HILLERTON, J.E.; BRANLEY, A.J. Variability between Muscidae population of dairy heifers on two different types of pasture in southern England. British Veterinary Journal, v.142, n.2, p.155-162, 1986. Disponível em: <http:// www.sciencedirect.com/science>. Acesso em 27 nov. 2009. doi:10.1016/0007-1935(86)90092-8.

HONER, M.R. et al. A mosca-dos-chifres: história, biologia e controle. Campo Grande, MS: Embrapa-CNPGC, 1991. 34p. (Documentos 45).

KEIDING, J. The house fly: biology and control. Geneva: WHO, 1986. (Vector Control Series 63).

LIMA, L.G.F. et al. Localização preferencial e índices diferenciados de infestação da mosca-dos-chifres (Haematobia irritans) em bovinos da raça Nelore. Pesquisa Veterinária Brasileira, v.22, n.1, p.25-32, 2002. Disponível em: <http:/ /www.scielo.br/pdf/pvb/v22n1/8868.pdf>. Acesso em 27 nov. 2009. doi: 10.1590/S0100-736X2002000300004.
LYSYK, T.J.; AXTELL, R.C. A simulation model of house fly (Diptera: Muscidae) development in poultry manure. Canadian Entomology, v.119, n.5, p.427-37, 1987.

LYSYK, T.J.; MOON, R.D. Diapause induction in the horn fly (Diptera: Muscidae). Environmental Entomology, v.28, p.387-397, 1994.

MARCHIORI, C.H. et al. Artrópodes associados a massas fecais bovinas no Sul do Estado de Goiás. Neotropical Entomology, v. 30, n. 1, p. 19-24, 2001. Disponível em: <http:// www.scielo.br/>. Acesso em 27 nov. 2009. doi: 10.1590/S1519566X2001000100004.

MENDES, J.; LINHARES, A.X. Atratividade por iscas, sazonalidade e desenvolvimento ovariano em várias espécies de Muscidae (Diptera). Revista Brasileira de Entomologia, v.37, p. 289-297, 1993.

MENDES, J.; LINHARES, A.X. Diapause, pupation, sites and parasitism of the hornfly, Haematobia irritans, in South-eastern Brazil. Medical and Veterinary Entomology, v.13, p.1-6, 1999.

MOON, R.D. Muscid flies (Muscidae). In: MULLEN, G.; DURDEN, L. Medical and veterinary entomology. Burlington: Academic, 2002. Cap.14, p.279-300.

NUORTEVA, P. Synantropy of blowflies (Diptera: Calliphoridae) in Finland. Annales Entomologici Fennici, v.29, p.1-49, 1963.

RIBEIRO, P.B. et al. Flutuação Populacional de Dermatobia hominis sobre bovinos no município de Pelotas, RS. Arquivo Brasileiro de Medicina Veterinária e Zootecnia, v.41, n.3, p.223- 231, 1989.

SCHREIBER, E.T.; CAMPBELL, J.B. Horn fly (Diptera: Muscidae) distribution on cattle as influenced by host color and time of day. Environmental Entomology, v.15, n.6, p.13071309, 1986.

THOMAS, D.; KUNZ, S.D. Diapause survival of overwintering populations of the horn fly Haematobia irritans (Diptera: Muscidae), in south-central Texas. Environmental Entomology, v.15, p.44-48, 1986.

ZUMPT, F. The Stomoxyine biting flies of the world. Stuttgart: G ustav Fischer Verlag, 1973. 175p. 